\title{
Correction to: Determinants of Influenza Mortality Trends: Age-Period-Cohort Analysis of Influenza Mortality in the United States, 1959-2016
}

\author{
Enrique Acosta ${ }^{1,2}$. Stacey A. Hallman ${ }^{3} \cdot$ Lisa Y. Dillon ${ }^{1} \cdot$ Nadine Ouellette $^{1}$. \\ Robert Bourbeau ${ }^{1}$ - D. Ann Herring ${ }^{4} \cdot K_{\text {Kris Inwood }}^{5}$ • David J. D. Earn ${ }^{6,7}$. \\ Joaquin Madrenas ${ }^{8} \cdot$ Matthew S. Miller $^{7,9,10}$. Alain Gagnon ${ }^{1,11}$
}

Published online: 28 October 2019

(C) Population Association of America 2019

\section{Correction to: Demography \\ https://doi.org/10.1007/s13524-019-00809-y}

The authors would like to correct the second sentence of abstract to read:

First, we use Lexis surfaces based on Serfling models to highlight influenza mortality patterns as well as to identify lingering effects of early-life exposure to specific influenza virus subtypes (e.g., H1N1, H3N2).

Publisher's Note Springer Nature remains neutral with regard to jurisdictional claims in published maps and institutional affiliations.

The online version of the original article can be found at https://doi.org/10.1007/s13524-019-00809-y

Alain Gagnon

alain.gagnon.4@umontreal.ca

Extended author information available on the last page of the article 


\section{Affiliations}

Enrique Acosta ${ }^{1,2} \cdot$ Stacey A. Hallman ${ }^{3} \cdot$ Lisa Y. Dillon ${ }^{1} \cdot$ Nadine Ouellette $^{1}$. Robert Bourbeau $^{1}$ - D. Ann Herring ${ }^{4} \cdot$ Kris Inwood $^{5}$ - David J. D. Earn ${ }^{6,7}$. Joaquin Madrenas ${ }^{8}$ - Matthew S. Miller ${ }^{7,9,10}$ - Alain Gagnon ${ }^{1,11}$

1 Département de Démographie, Université de Montréal, C.P. 6128, succursale Centre-ville, Montréal, QC H3C 3J7, Canada

2 Max Planck Institute for Demographic Research, Rostock, Germany

3 Demography Division, Statistics Canada, Ottawa, Canada

4 Department of Anthropology, McMaster University, Hamilton, Canada

5 Department of History, University of Guelph, Guelph, Canada

6 Department of Mathematics and Statistics, McMaster University, Hamilton, Canada

7 Michael G. DeGroote Institute for Infectious Diseases Research, McMaster University, Hamilton, Canada

8 Los Angeles Biomedical Research Institute, Harbor-UCLA Medical Center, Torrance, CA, USA

9 Department of Biochemistry and Biomedical Sciences, McMaster, Hamilton, Canada

10 McMaster Immunology Research Centre, McMaster University, Hamilton, Canada

11 Public Health Research Institute (IRSPUM), Université de Montréal, Montreal, Canada 